\title{
以三氟乙酸及其衍生物为三氟甲基源的三氟甲基化反应研究进展
}

\author{
季小明 ${ }^{a}$ 史广法 ${ }^{a}$ 张扬会*,a,b \\ $\left({ }^{a}\right.$ 同济大学化学科学与工程学院 上海 200092) \\ $\left({ }^{b}\right.$ 上海市化学品分析、风险评估与控制重点实验室＼cjkstart同济大学上海 200092)
}

\begin{abstract}
摘要 含有三氟甲基基团的有机分子中常常表现出独特的物理和化学性质, 具有非常广泛的应用. 三氟乙酸及其衍生 物具有廉价、易得、稳定等优点, 并且反应的副产物是二氧化碳，是理想的三氟甲基化试剂，具有广阔的应用前景. 综 述了以三氟乙酸及其衍生物为三氟甲基源的三氟甲基化反应的最新研究进展, 主要包括 $\mathrm{C}-\mathrm{X}(\mathrm{X}=\mathrm{Br}, \mathrm{I})$ 键、 $\mathrm{C}-\mathrm{H}$ 键, $\mathrm{C}=\mathrm{X}(\mathrm{X}=\mathrm{C}, \mathrm{O})$ 的三氟甲基化反应，对反应的相关机理进行了论述.

关键词 三氟乙酸; 氟化学; 氟; 三氟乙基化; 碳氢键活化
\end{abstract}

\section{Progress of Trifluoromethylation Using Trifluoroacetic Acid and Its Derivatives as $\mathrm{CF}_{3}$-Sources}

\author{
$\mathrm{Ji}_{\text {, Xiaoming }}{ }^{a} \quad$ Shi, Guangfa ${ }^{a} \quad$ Zhang, Yanghui ${ }^{*}, a, b$ \\ $\left({ }^{a}\right.$ School of Chemical Science and Engineering, Tongji University, Shanghai 200092) \\ $\left({ }^{b}\right.$ Shanghai Key Laboratory of Chemical Assessment and Sustainability, Tongji University, Shanghai 200092)
}

\begin{abstract}
Organic molecules containing trifluoromethyl groups often exhibit unique physical and chemical properties, and have found extensive applications. Trifluoroacetic acid and its derivatives have advantages including low cost, ready availability and high stability. Furthermore, $\mathrm{CO}_{2}$ is the byproduct. Therefore, trifluoroacetic acid and its derivatives are ideal trifluoromethylating reagents, and have great application potentials. The progress of trifluoromethylation reactions using trifluoroacetic acid and its derivatives as $\mathrm{CF}_{3}$-sources is reviewed, including the reactions of $\mathrm{C}-\mathrm{X}(\mathrm{X}=\mathrm{Br}$ and $\mathrm{I}), \mathrm{C}-\mathrm{H}$, and $\mathrm{C}=\mathrm{X}(\mathrm{X}=\mathrm{C}$, O). The mechanisms are also introduced.
\end{abstract}

Keywords trifluoroacetic acid; $\mathrm{CF}_{3}$-sources; trifluoromethylation reaction; $\mathrm{C}-\mathrm{H}$ activation

含氟基团具有独特的性质，这类基团广泛存在于医 药 ${ }^{[1]}$ 、农药 ${ }^{[2]}$ 和材料 ${ }^{[3]}$ 中. 市场销售的大约 $20 \%$ 的医药和 $30 \% \sim 40 \%$ 的农药中含有氟原子, 销量排名前 10 的 4 种 药物中含有氟原子 ${ }^{[4]}$. 与不含氟药物相比, 含氟药物通 常具有代谢稳定、药物代谢动力学高等特性 ${ }^{[4 a, 5]}$. 在含氟 基团中，三氟甲基基团是一种重要的结构单元，不仅可 以用于染料、高分子材料的改性, 也可以调节药物的代 谢活性, 提高脂溶性、生物渗透性和靶向选择性等, 在 改善药效和药物动力学方面具有重要作用 ${ }^{[6]}$. 大量已经 上市或处于临床试验中的药物含有三氟甲基, 表现出了 相较于其他药物更好的疗效. 例如, 用于治疗重度抑郁 症、神经性贪食、强迫性神经失调的药物 Fluoxetine 与 传统抗忧郁药物相比副作用较小 ${ }^{[6 a]}$; 新一代非甾体抗
炎镇痛药 celecoxib 用于治疗多种临床常见的急性疼痛、 术后疼痛等，也用于治疗慢性疼痛，如骨关节炎、类风 湿关节炎、强直性脊柱炎等 ${ }^{[7]}$; enzalutamide 被认为是最 好的一个治疗转移性前列腺癌的药物 ${ }^{[6]}$. 此外, 还有其 他一些含三氟甲基的上市的畅销药，包括 dutasteride、

bicalutamide、leflunomide、nilutamide、dexiansoprazole 等 ${ }^{[6,6 f]}$. 不仅许多医药中含有三氟甲基基团, 很多农药 中也含有三氟甲基基团。例如，除草剂氟乐灵 (Trifluralin)、吡氟禾草灵(Fluazifop-butyl)、乙氧氟草醚 (Oxyfluorfen) 和杀虫剂氟虫腈 (Fipronil), 氟啶腿 (Chlorfluazuron) 以及杀菌剂啶氧菌酯(Picoxystrobin)等. 这些含三氟甲基农药在农业生产中发挥了非常重要的 作用.

\footnotetext{
* Corresponding author. E-mail: zhangyanghui@tongji.edu.cn

Received October 25, 2018; revised December 6, 2018; published online December 21, 2018.

Project supported by the National Natural Science Foundation of China (Nos. 21372176, 21672162).

国家自然科学基金(Nos. 21372176, 21672162)资助项目.
} 
在有机分子中引入三氟甲基基团, 能够使其表现出 独特的物理和化学性质, 具有重要的意义 ${ }^{[8]}$. 因此, 三 氟甲基的引入受到了科学工作者的广泛关注, 并且发展 出了一系列引入三氟甲基的方法, 包括钯 ${ }^{[9]}$ 、铜 ${ }^{[10]}$ 及其 他过渡金属催化的三氟甲基化 ${ }^{[11 ~ 14]}$ 或者自由基三氟甲 基化等方法 ${ }^{[15,16]}$. 新的三氟甲基化试剂的发现加速了三 氟甲基化反应的发展 ${ }^{[17]}$. 三氟甲基化试剂一般可以分 为亲电、亲核、自由基三种类型 ${ }^{[18]}$. 尽管这些三氟甲基 化试剂可以用于有机分子的三氟甲基化反应，但在使用 过程中存在许多缺点. 例如, 有些三氟甲基化试剂价格 昂贵、毒性强、使用不方便, 有些试剂现在还没有商业 化或者使用过程中会产生大量的化学废弃物. 因此, 为 了解决上述三氟甲基化试剂的诸多缺点, 迫切需要开发 出廉价且易于处理的新的三氟甲基化试剂.

三氟乙酸及其衍生物作为一种具有广阔前景的三 氟甲基化试剂, 具有廉价、易得、后处理方便等优点, 并 且反应的副产物是二氧化碳, 符合绿色化学理念 ${ }^{[19]}$. 本 文将系统总结利用三氟乙酸及其衍生物为三氟甲基源 来实现三氟甲基化反应的研究进展.

\section{$1 \mathrm{C}$ - $\mathrm{X}(\mathrm{X}=\mathrm{Br}, \mathrm{I})$ 键的三氟甲基化反应}

含三氟甲基的芳烃是一类重要的结构单元 ${ }^{[20]}$, 因 此, 在芳环的特定位置上引入三氟甲基基团具有重要的 意义. 早在 1981 年, Kondo 课题组 ${ }^{[21]}$ 以 $\mathrm{CF}_{3} \mathrm{CO}_{2} \mathrm{Na}$ 为三 氟甲基源, $\mathrm{CuI}$ 为催化剂, $N$-甲基吡咯烷酮(NMP)为溶剂, 在氩气氛围下实现了碘苯 $\mathrm{C}-\mathrm{X}(\mathrm{Br}, \mathrm{I})$ 键的三氟甲基化 反应(Scheme 1). 与之前报道的方法相比, 该方法操作 简单、三氟甲基源来源丰富，产率中等至良好.

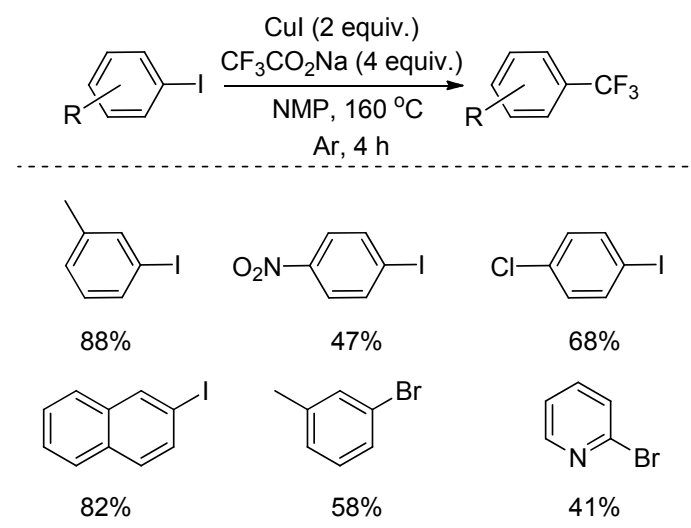

图式 1 卤代芳烃与三氟乙酸钠的三氟甲基化反应 Scheme 1 Trifluoromethylation of aromatic halides with sodium trifluoroacetate

1998 年, $\mathrm{Hünig}$ 小组 ${ }^{[22]}$ 以 $\mathrm{CF}_{3} \mathrm{CO}_{2} \mathrm{Na}$ 为三氟甲基源, $\mathrm{CuI}$ 为催化剂, $N, N-$ 二甲基乙酰胺 (DMA)与 $\mathrm{PhMe}$ 的混合 物为溶剂，实现了多取代碘代芳烃的三氟甲基化反应
(Scheme 2).

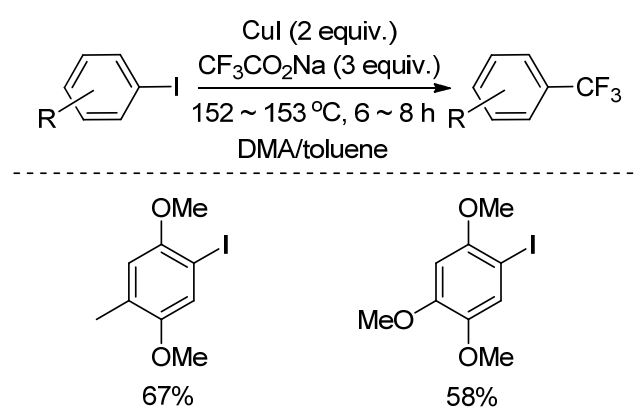

图式 2 多取代卤代芳烃与三氟乙酸钠的三氟甲基化反应 Scheme 2 Trifluoromethylation of multisubstituted aromatic halides with sodium trifluoroacetate

2001 年, Branch 小组 ${ }^{[23]}$ 以 $\mathrm{CF}_{3} \mathrm{CO}_{2} \mathrm{~K}$ 为三氟甲基源, $\mathrm{CuI}$ 为催化剂, $N, N-$ 二甲基甲酰胺 (DMF) 与 $\mathrm{PhMe}$ 的混合 物为溶剂, $110{ }^{\circ} \mathrm{C}$ 下反应 $1.5 \mathrm{~h}$, 实现了 5-溴代异吲哚碳 澳键的三氟甲基化反应(Scheme 3), 该反应产率较高.

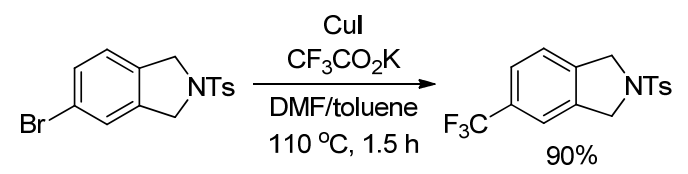

图式 3 -溴代异吲哚与三氟乙酸钠的三氟甲基化反应 Scheme 3 Trifluoromethylation of 5-bromoisoindole with sodium trifluoroacetate

2007 年, Langlois 课题组 ${ }^{[24]}$ 报道了芳基卤代物与三 氟乙酸甲酯(MTFA)的亲核三氟甲基化反应(Scheme 4). 该反应中以三氟乙酸甲酯为三氟甲基源, 芳基碘代物或 芳基溴代物都能发生反应，而芳基氯代物几乎不能发生 反应. 以 $\mathrm{DMF}$ 为溶剂时, 反应温度需要加热到 $180{ }^{\circ} \mathrm{C}$; 而以环丁砜为溶剂时, 反应在 $140{ }^{\circ} \mathrm{C}$ 时就能很好地进 行, 这可能是由于环丁砜对三氟甲磺酸亚铜中间体具有 配位作用. 无论以 DMF 为溶剂还是以环丁砜为溶剂, 都需要加入过量的碘化亚铜, 这是由于氧化加成的速率 远小于脱羧的速率，脱羧产生的不稳定的三氟甲基负离 子需要铜来稳定.

2010 年, Vicic 小组 ${ }^{[25]}$ 首次以合成的三氟乙酸 $N$-杂 环卡宾(NHC)铜配合物作为催化剂和三氟甲基源, 实现 了碘苯或溴苯的碳卤键三氟甲基化反应(Scheme 5). 当 以 $\left(\mathrm{SI}{ }^{i} \mathrm{Pr}\right) \mathrm{Cu}(\mathrm{TFA})$ 为催化剂及三氟甲基源, 碘苯既作溶 剂又作反应物，在 $160{ }^{\circ} \mathrm{C}$ 时反应得到中等收率的三氟 甲基化产物; 而以 $\mathrm{CuI}$ 为催化剂, $\mathrm{CF}_{3} \mathrm{CO}_{2} \mathrm{Na}$ 为三氟甲基 源时，以碘苯既作溶剂又作反应物，得不到三氟甲基化 的产物. 对比试验表明，在芳基卤溶剂中，铜配合物的 效果要好于无配体的 CuI. 当把卤苯溶剂与 $N, N$-二甲基 乙酰胺按体积比配成 $1: 1$ 时, 上述含三氟甲基铜配合 
物发生三氟甲基化的效果要差于 $\mathrm{CuI} / \mathrm{CF}_{3} \mathrm{CO}_{2} \mathrm{Na}$ 体系, 该现象说明了基于胺的溶剂可能对 $\mathrm{CuI}$ 有配位作用, 加 速了脱羧三氟甲基化反应的进程.

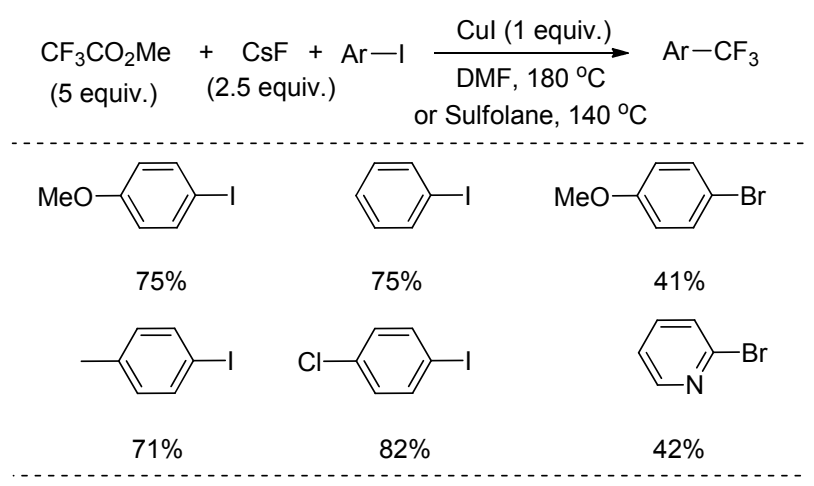

Possible reaction mechanism

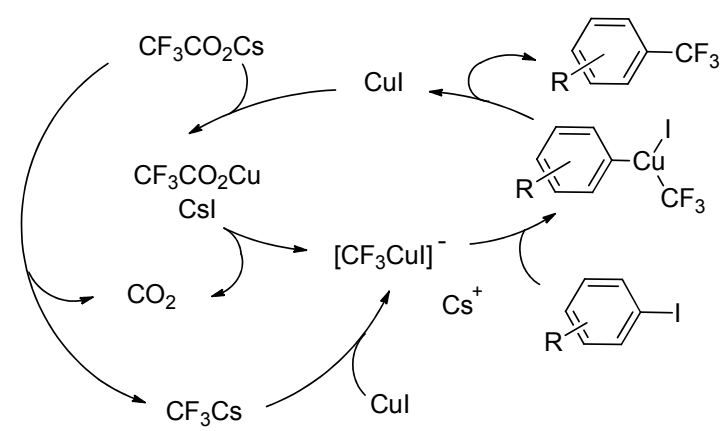

图式 4 卤代芳烃与三氟乙酸甲酯的三氟甲基化反应

Scheme 4 Trifluoromethylation of aromatic halides with methyl trifluoroacetate

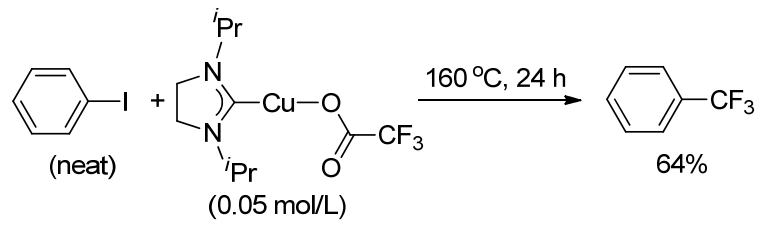

图式 5 卤代芳烃的脱羧三氟甲基化反应

Scheme 5 Decarboxylative trifluoromethylation of aromatic halides

2011 年, Duan 和 Li 课题组 ${ }^{[26]}$ 报道了铜催化的碘苯 与 $\mathrm{CF}_{3} \mathrm{CO}_{2} \mathrm{Na}$ 的三氟甲基化反应(Scheme 6). 该反应以 对氯碘苯为原料, $\mathrm{Cu}$ 粉为催化剂, $\mathrm{CF}_{3} \mathrm{CO}_{2} \mathrm{Na}$ 为三氟甲 基源, $\mathrm{Ag}_{2} \mathrm{O}$ 为添加剂, $\mathrm{DMF}$ 为溶剂, $130{ }^{\circ} \mathrm{C}$ 下反应 $15 \mathrm{~h}$, 得到 $61 \%$ 的产率. 该反应具有较好的官能团兼容性, 含 有卤素、氰基、酯基、硝基等基团的底物都能很好地发 生反应，产率中等至优秀. 反应条件与之前报道的以 $\mathrm{CF}_{3} \mathrm{CO}_{2} \mathrm{Na}$ 为三氟甲基源的反应相比，条件较温和.

2012 年, $\mathrm{Wu}$ 小组 ${ }^{[27]}$ 以碘苯或溴苯为底物, $\mathrm{CuI}$ 为催 化剂，1,10-菲啰啉为配体，铯盐为碱，廉价且易得的三 氟乙酸甲酯(MTFA)为三氟甲基源, DMF 为溶剂, 首次 实现了卤苯与 MTFA 的三氟甲基化反应(Scheme 7). 该

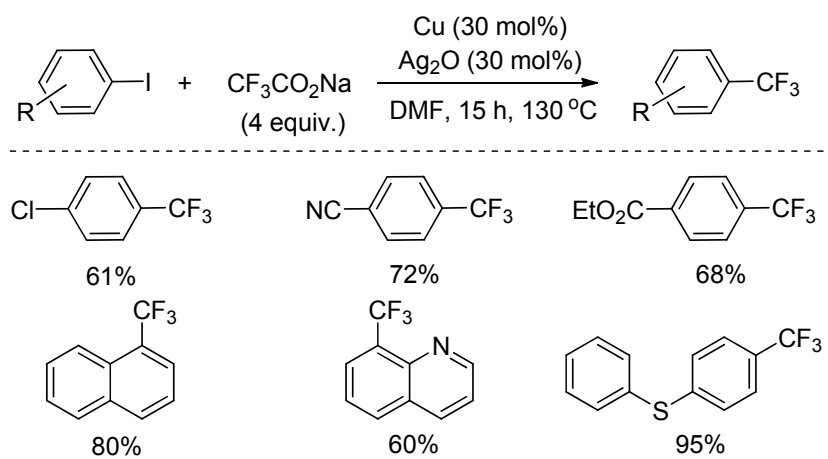

图式 6 铜催化的卤代芳烃与三氟乙酸钠的三氟甲基化反应 Scheme 6 Copper-catalyzed trifluoromethylation of aryliodides with sodium trifluoroacetate

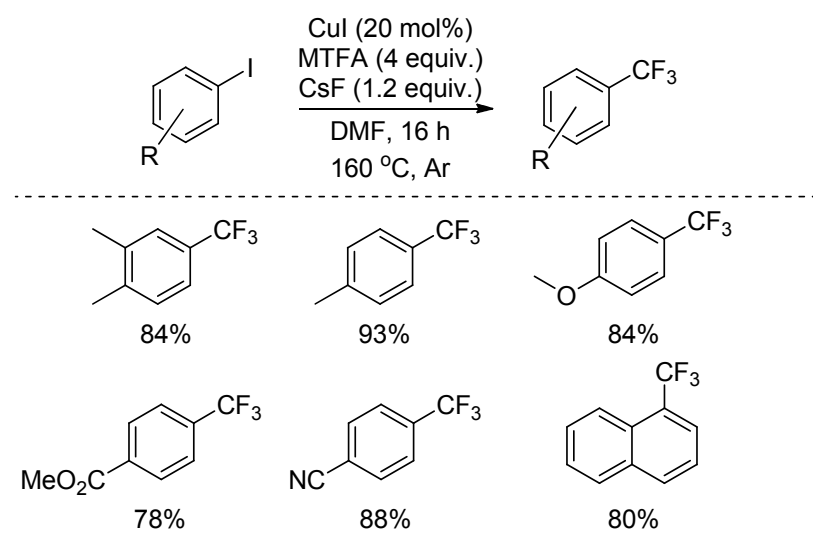

Possible reaction mechanism

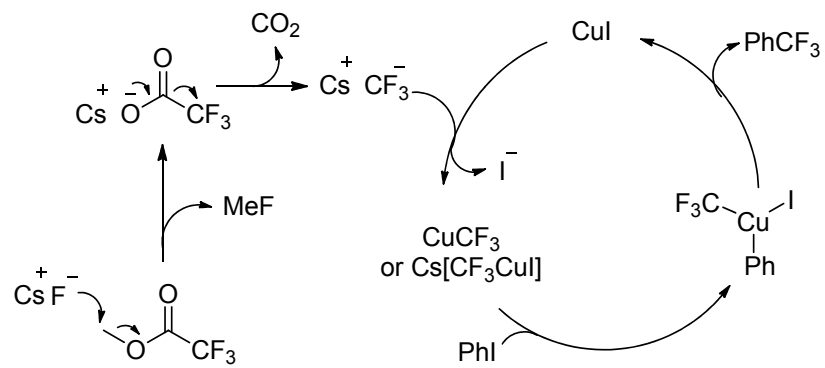

图式 7 铜催化的芳基卤与三氟乙酸甲酯的三氟甲基化反应 Scheme 7 Copper-catalyzed trifluoromethylation of aryl halides with methyl trifluoroacetate

反应中, 铯盐对于 MTFA 的脱羧产生三氟甲基负离子起 了至关重要的作用.

2013 年, Buchwald 课题组 ${ }^{[28]}$ 首次实现了在流动体 系中 $\mathrm{CuI}$ 促进的碘代芳烃或杂芳烃的碳碘键的三氟甲基 化反应(Scheme 8). 该反应是在微型反应器中进行的, 以 $\mathrm{CF}_{3} \mathrm{CO}_{2} \mathrm{~K}$ 为三氟甲基源，吡啶为配体， NMP 为溶剂, $200{ }^{\circ} \mathrm{C}$ 下反应 $16 \mathrm{~min}$, 产率较高且可以放大到克级规 模. 该反应时间短，官能团兼容性好，含有硝基、磺酰胺 基、吡啶、吲哚、吡唑等单元的底物也能很好地发生反 应.

2014 年, Sanford 课题组 ${ }^{[29]}$ 以三氟乙酸苯酯为三氟 
甲基源, 详细研究了钯催化脱羧三氟甲基化反应的催化 循环(Scheme 9). 作者研究了催化循环的四个基本步骤: 首先, (Cp)Pd(allyl)与 RuPhos 在 $60{ }^{\circ} \mathrm{C}$ 时反应 $30 \mathrm{~min}$, 生 成的(RuPhos)nPd ${ }^{0}$ 中间体与三氟乙酸苯酯发生氧化加 成, 再与三氟乙酸发生配体交换生成 (RuPhos)Pd$\left(\mathrm{COCF}_{3}\right)\left(\mathrm{CO}_{2} \mathrm{CF}_{3}\right)$; 然后将( RuPhos) $\mathrm{Pd}\left(\mathrm{COCF}_{3}\right)\left(\mathrm{CO}_{2} \mathrm{CF}_{3}\right)$ 在苯中 $80{ }^{\circ} \mathrm{C}$ 回流 $1.5 \mathrm{~h}$ 脱去 $\mathrm{CO}$ 生成中间体(RuPhos)$\operatorname{Pd}\left(\mathrm{CF}_{3}\right)\left(\mathrm{CO}_{2} \mathrm{CF}_{3}\right)$; 接着, (RuPhos) $\mathrm{Pd}\left(\mathrm{CF}_{3}\right)\left(\mathrm{CO}_{2} \mathrm{CF}_{3}\right)$ 与 $\mathrm{Zn}(\mathrm{Aryl})_{2}$ 发生转金属化生成(RuPhos) Pd $\left(\mathrm{CF}_{3}\right)(\operatorname{aryl})$; 最 后, $(\mathrm{RuPhos}) \mathrm{Pd}\left(\mathrm{CF}_{3}\right)$ (aryl) 在苯中 $90{ }^{\circ} \mathrm{C}$ 加热 $12 \mathrm{~h}$ 发生还 原消除生成三氟甲基化的芳基产物. 与其他两个步骤相 比, 脱去 $\mathrm{CO}$ 和还原消除需要相对较高的温度. 这一反 应说明, 将三氟乙酸酯用于交叉偶联反应中具有潜在的 可能.

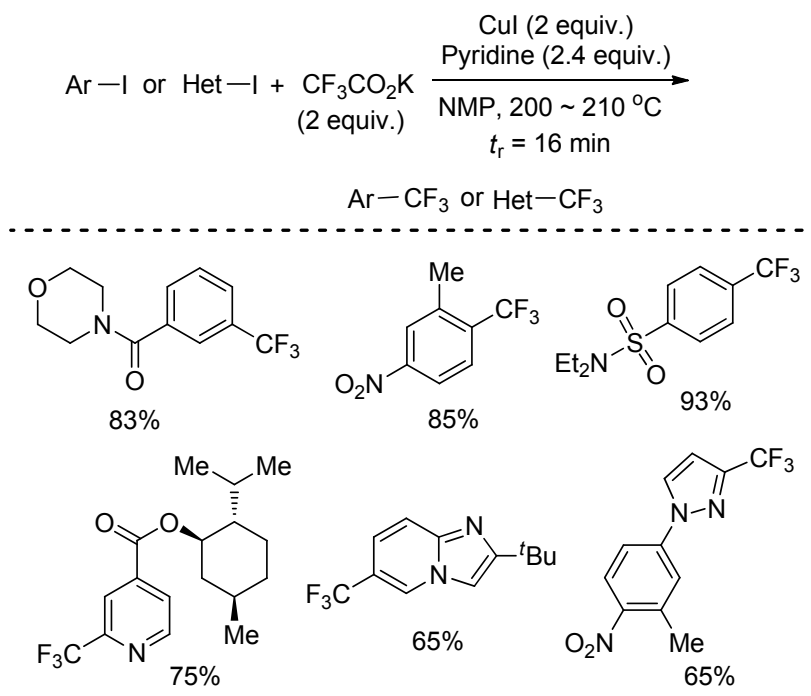

图式 8 铜催化的碘代芳烃与三氟乙酸钾的三氟甲基化反应 Scheme 8 Copper-catalyzed trifluoromethylation of aryliodides with potassium trifluoroacetate

2016 年, Weng 等 ${ }^{[30]}$ 以新合成的廉价的 $\left[\mathrm{Cu}\left(\mathrm{O}_{2} \mathrm{C}-\right.\right.$ $\mathrm{CF}_{3}$ )(phen)] 络合物为三氟甲基源, $\mathrm{NaF}$ 为添加剂, $\mathrm{DMF}$ 为溶剂, $140{ }^{\circ} \mathrm{C}$ 下反应, 实现了卤代芳烃或杂芳烃的三 氟甲基化反应(Scheme 10). 该反应具有良好的官能团 容忍性, 含有醛基、乙酰基、硝基、吡啶等基团的底物 反应得到中等至良好的收率，该方法也可以用于雌二醇 衍生物的三氟甲基化反应. 机理研究表明, 该反应经历 了亲核反应的过程而不是自由基的反应过程.

2017 年, Torikai 小组 ${ }^{[31]}$ 以 $\mathrm{CF}_{3} \mathrm{CO}_{2} \mathrm{Na}$ 为三氟甲基源, $\mathrm{CuI}$ 为催化剂, $\mathrm{DMF}$ 为溶剂, 在微波辐射下实现了溴代 苯并鲐啶的三氟甲基化反应(Scheme 11). 该反应无需加 入配体, 即使两个溴取代的底物也能发生双三氟甲基化 反应. 而在油浴条件下, 在 NMP 中回流 $10.5 \mathrm{~h}$, 该三氟 甲基化反应不能发生.

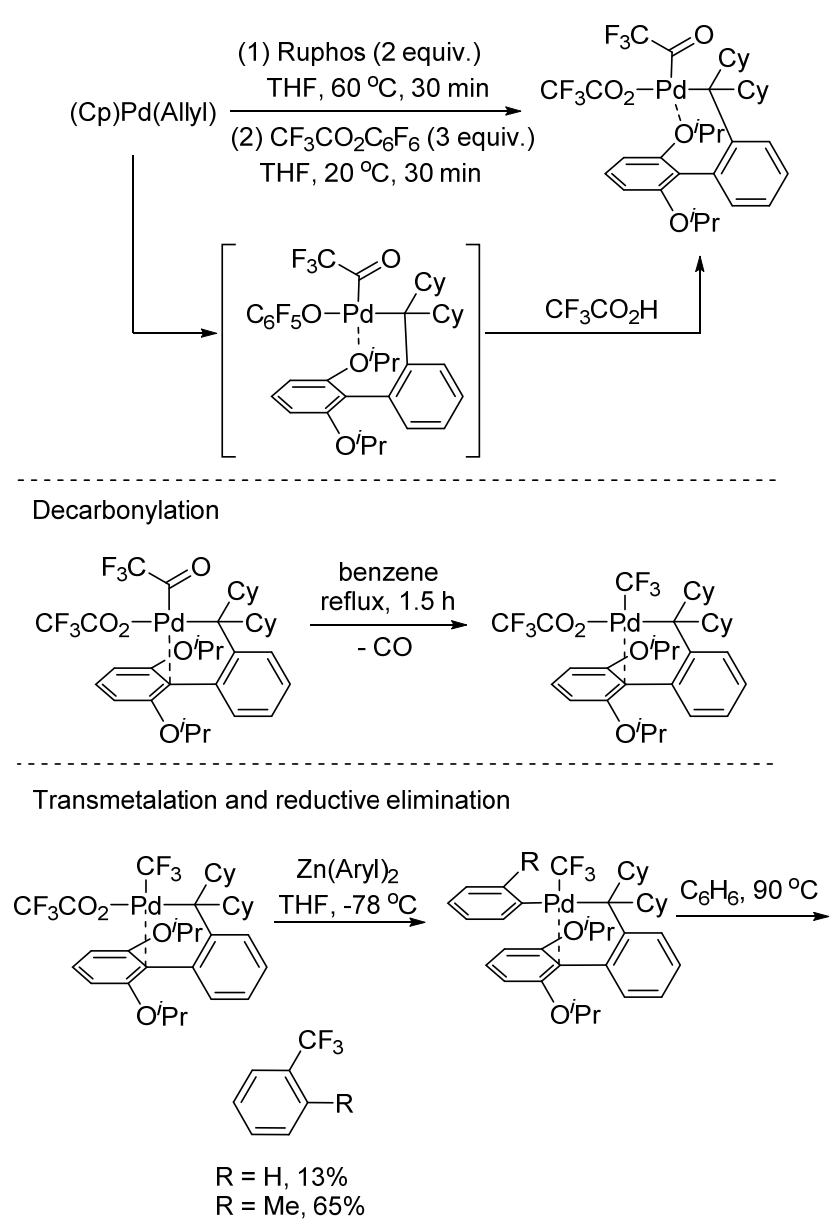

图式 9 钯催化的脱羧三氟甲基化反应的催化循环

Scheme 9 Catalytic cycle for Palladium-catalyzed decarbonylative trifluoromethylation

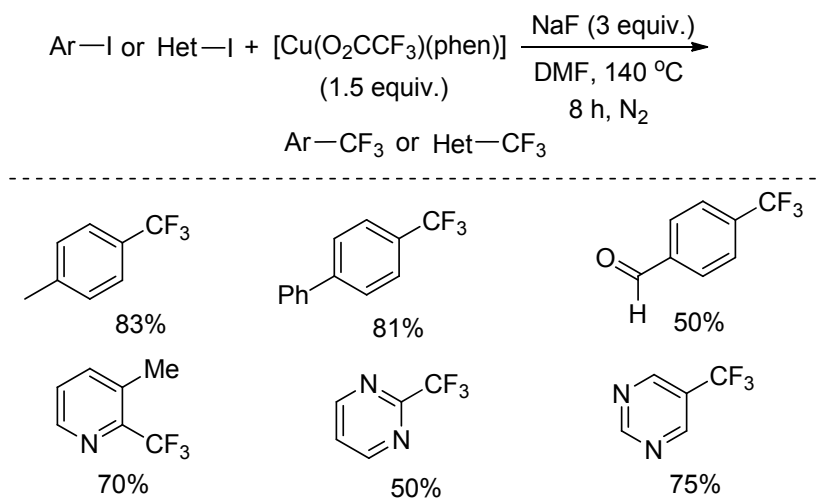

图式 10 以 $\left[\mathrm{Cu}\left(\mathrm{O}_{2} \mathrm{CCF}_{3}\right)\right.$ (phen)]为三氟甲基化试剂的反应 Scheme 10 Trifluoromethylation using $\left[\mathrm{Cu}\left(\mathrm{O}_{2} \mathrm{CCF}_{3}\right)(\right.$ phen) $]$ as trifluoromethylating reagent

\section{$2 \mathrm{C}-\mathrm{H}$ 键的三氟甲基化反应}

$\mathrm{C}-\mathrm{H}$ 键官能团化反应具有较高的原子经济性和步 骤经济性的特点, 符合绿色化学发展理念 ${ }^{[32]}$. 因此, 直 


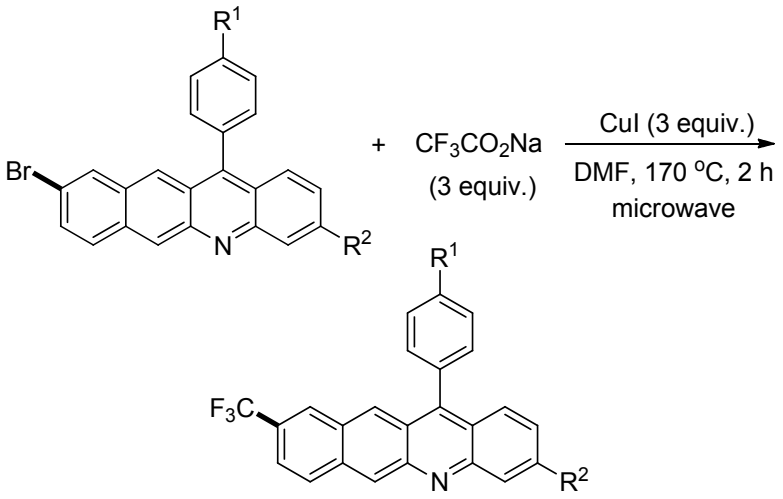<smiles>FC(F)(F)c1ccc2cc3nc4ccccc4c(-c4ccccc4)c3cc2c1</smiles>

$41 \%$<smiles>COc1ccc2c(-c3ccc(C(F)(F)F)cc3)c3cc4cc(C(F)(F)F)ccc4cc3nc2c1</smiles>

$76 \%$<smiles>FC(F)(F)c1ccc(-c2c3ccccc3nc3cc4ccc(C(F)(F)F)cc4cc23)cc1</smiles>

$69 \%$<smiles>FC(F)(F)c1ccc(-c2c3ccc(C(F)(F)F)cc3nc3cc4ccc(C(F)(F)F)cc4cc23)cc1</smiles>

$10 \%$
图式 11 微波辐射下苯并呋啶衍生物的三氟甲基化反应 Scheme 11 Trifluoromethylation of phenyl benzoacridine derivative under microwave irradiation

接的 $\mathrm{C}-\mathrm{H}$ 键三氟甲基化反应是合成含三氟甲基化合物 最简便的方法之一. 早在 1978 年, Vassiliev 等 ${ }^{[33]}$ 通过电 解 $\mathrm{CF}_{3} \mathrm{CO}_{2} \mathrm{H}$ 与 $\mathrm{CH}_{3} \mathrm{CN}$ 混合溶液的方法, 在阳极脱羧产 生三氟甲基自由基, 实现了苯环的 $\mathrm{C}-\mathrm{H}$ 键三氟甲基化 反应(Scheme 12). 在该反应中, $\mathrm{CH}_{3} \mathrm{CN}$ 对三氟甲基自由 基的产生至关重要, 在体系中不加入 $\mathrm{CH}_{3} \mathrm{CN}$, 生成的是 三氟乙酰氧基取代的产物.

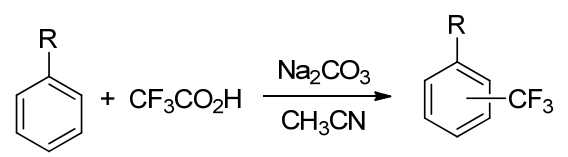

图式 12 苯衍生物的三氟甲基化反应

Scheme 12 Trifluoromethylation of benzene derivatives

1990 年, Yoshida 等 ${ }^{[34]}$ 以二(三氟乙酰基)过氧化物为 三氟甲基源, 实现了苯环、呋喃环、噻吩环的 $\mathrm{C}-\mathrm{H}$ 键 三氟甲基化反应(Scheme 13). 该反应无需加入金属催 化剂, 二(三氟乙酰基)过氧化物在加热条件下就可以分 解产生三氟甲基自由基. 机理研究表明, 该反应是通过 从底物到过氧化物的电子转移进行的, 所以电子转移过 程是该反应的决速步骤. 含苯环底物的存在加速了过氧
化物分解产生三氟甲基自由基的速率.

$$
\begin{aligned}
& \mathrm{Ar}-\mathrm{I} \text { or } \mathrm{Het}-\mathrm{I}+\left(\mathrm{CF}_{3} \mathrm{CO}_{2}\right)_{2} \stackrel{60 \text { or } 70{ }^{\circ} \mathrm{C}}{\longrightarrow} \mathrm{Ar}-\mathrm{CF}_{3} \text { or } \mathrm{Het}-\mathrm{CF}_{3} \\
& (1.5 \sim 2 \text { equiv. })
\end{aligned}
$$

图式 13 芳烃与三氟乙酸过氧化物的三氟甲基化反应 Scheme 13 Trifluoromethylation of aromatic compounds with bis(trifluoroacetyl)peroxide

1993 年, Mallouk 课题组 ${ }^{[35]}$ 以粉末状的 $\mathrm{TiO}_{2}$ 为光催 化剂, $\mathrm{CF}_{3} \mathrm{CO}_{2} \mathrm{Ag}$ 为三氟甲基源, $\mathrm{MeCN}$ 为溶剂, 实现了 苯环 $\mathrm{C}-\mathrm{H}$ 键三氟甲基化反应(Scheme 14). 在该反应中, 生成的银单质附着在光催化剂 $\mathrm{TiO}_{2}$ 表面, 需要加入大 大过量的 $\mathrm{TiO}_{2}$, 产物的产率较低, 区域选择性较差. 反 应同时生成了由三氟甲基自由基二聚产生的六氟乙烷, 并且往体系中加入自由基捕捉剂，三氟甲基化反应被完 全抑制，说明该反应经历了三氟甲基自由基中间体的过 程.

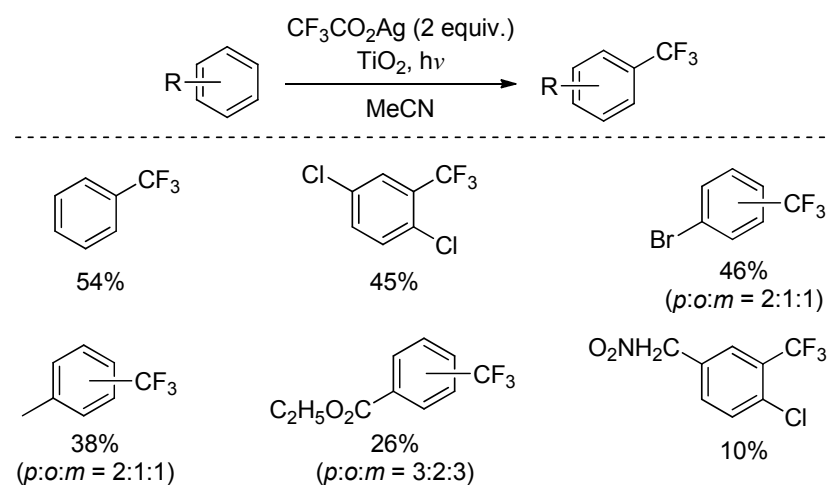

图式 14 芳烃的光化学三氟甲基化反应

Scheme 14 Photochemical trifluoromethylation of aromatic compounds

1995 年, Matsui 小组 ${ }^{[36]}$ 以三氟乙酸过氧化物 $\left(\left(\mathrm{CF}_{3} \mathrm{CO}_{2}\right)_{2}\right)$ 为三氟甲基源，二氯甲烷 $(\mathrm{DCM})$ 为溶剂, $0{ }^{\circ} \mathrm{C}$ 下应 $4 \mathrm{~h}$, 实现了 1,4 -萘醌的碳氢键三氟甲基化 反应(Scheme 15). 该反应经历了自由基的反应历程, 产 率较低.

1998 年, Matsuo 等 ${ }^{[37]}$ 基于已报道的 $\mathrm{XeF}_{2}$ 与 $\mathrm{CF}_{3} \mathrm{CO}_{2} \mathrm{H}$ 反应生成六氟乙烷的反应, 以二氯甲烷为溶 剂，往体系中加入含吸电子基团的芳烃，室温反应得到 中等收率的三氟甲基化的产物(Scheme 16). 该反应中, 三氟甲基化的区域选择性较差，富电子的苯甲醚和甲苯 
主要发生单氟取代的反应.<smiles>[R]C1=CC(=O)c2ccccc2C1=O</smiles><smiles>CC1=C(C(F)(F)F)C(=O)c2ccccc2C1=O</smiles><smiles>O=C1C(Nc2ccc(Cl)cc2)=C(C(F)(F)F)C(=O)c2ccccc21</smiles>

图式 15 菜醌的三氟甲基化反应

Scheme 15 Trifluoromethylation of naphthoquinones $\mathrm{Ar}-\mathrm{H}$ or $\mathrm{Het}-\mathrm{H} \underset{\text { r.t. }}{\stackrel{\mathrm{CF}_{3} \mathrm{CO}_{2} \mathrm{H}(3 \text { equiv. })}{\longrightarrow}} \mathrm{Ar}-\mathrm{CF}_{3}$ or Het $-\mathrm{CF}_{3}$<smiles>FC(F)(F)c1cc(Cl)ccc1Cl</smiles><smiles>COc1cc([N+](=O)[O-])cc(C(F)(F)F)c1OC</smiles>

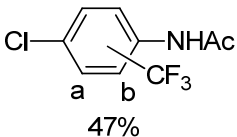
$a: b=1.5: 1$<smiles>CC(=O)OCC1OC(n2cc(C(F)(F)F)c(=O)[nH]c2=O)CC1OC(C)=O</smiles>

图式 16 二氟化氙促进的芳烃的三氟甲基化反应 Scheme 16 Trifluoromethylation of Aromatics with trifluoroacetic acid mediated by xenon difluoride

1999 年, Trevin 小组 ${ }^{[38]}$ 通过 Kolbe 电解氧化 $\mathrm{CF}_{3} \mathrm{CO}_{2} \mathrm{H}$ 产生三氟甲基自由基, 实现了 $\mathrm{PhCN}$ 的 $\mathrm{C}-\mathrm{H}$ 键三氟甲基化反应(Scheme 17). 该反应以 $\mathrm{CH}_{3} \mathrm{CN}$ 为溶 剂, 现场生成的三氟乙酸钠或三氟乙酸吡啶盐为三氟甲 基源，在小试和中试规模下进行了实验，产率中等，产 物的选择性分别为 $(o: m: p=46: 18: 36)$, 与非电解 反应的区域选择性一致. 在该试验中, 有机碱吡啶的效 果要好于无机碱氢氧化钠, 体系中吡啶的浓度对三氟甲 基化的产物产率影响较大, 可能是由于碱浓度的增加促 进了三氟乙酸在阳极表面的吸收. 较低的电流强度能避 免产物进一步发生三氟甲基化, $\mathrm{PhCN}$ 浓度越低, 产物 产率越高. 在最优条件下, 带吸电子基团的苯甲醛和苯 乙酮反应得到 35\%的产率, 硝基苯反应得到的产率较 低，而带供电子基团的甲苯不能发生三氟甲基化反应， 生成的是三氟乙酰氧基取代的甲苯.

2014 年, 我们课题组 ${ }^{[18]}$ 以 $\mathrm{CF}_{3} \mathrm{CO}_{2} \mathrm{H}$ 为三氟甲基源, $\mathrm{Ag}_{2} \mathrm{CO}_{3}$ 为催化剂, $\mathrm{Na}_{2} \mathrm{CO}_{3}$ 为碱, $\mathrm{MeCN}$ 或 $\mathrm{DCM}$ 为溶剂, $120{ }^{\circ} \mathrm{C}$ 下反应 $10 \mathrm{~h}$, 实现了缺电子苯环的 $\mathrm{C}-\mathrm{H}$ 键三氟

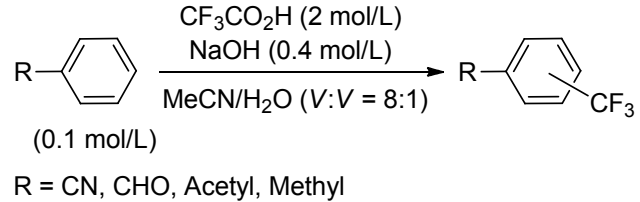

图式 17 通过 Kolbe 电解法进行的芳烃的三氟甲基化反应 Scheme 17 Trifluoromethylation of aromatic compounds via Kolbe electrolysis

甲基化反应(Scheme 18). 该反应体系能够容忍三氟甲 基、㲵基、酯基等基团，产率中等至良好. 在该反应中， 往体系中加入自由基捕捉剂 2,2,6,6-四甲基哌啶氧化物 (TEMPO)，三氟甲基化反应被完全抑制，说明了该反应 经历了自由基的历程.

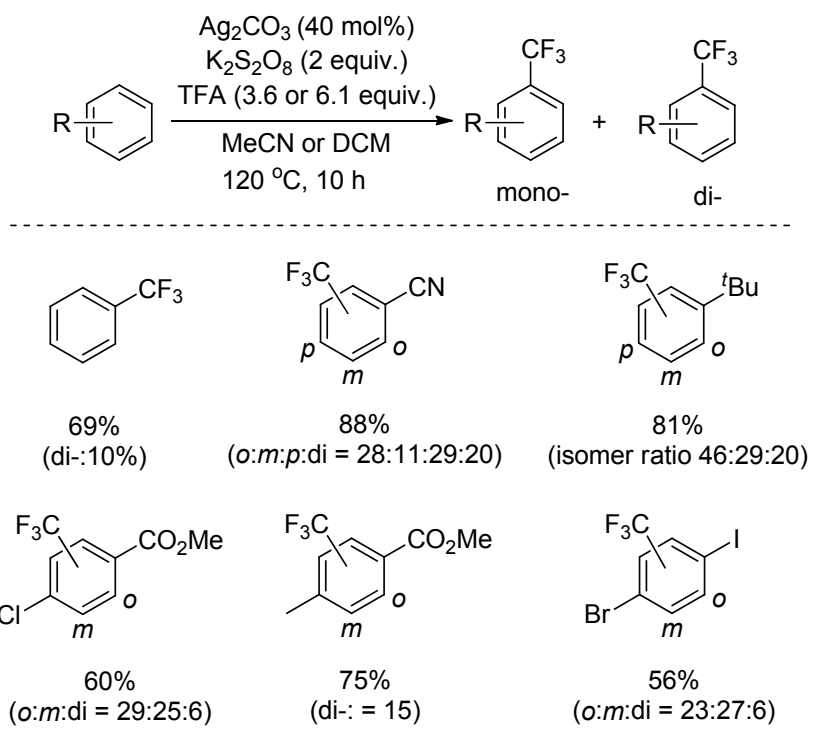

Possible reaction mechanism

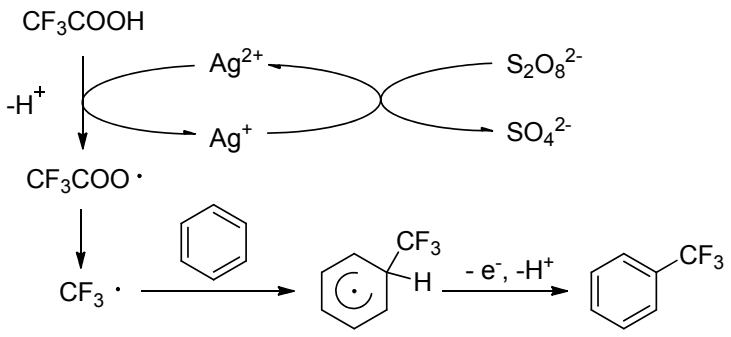

图式 18 以三氟乙酸为三氟甲基化试剂的反应

Scheme 18 Trifluoromethylation of arenes using trifluoroacetic acid as the trifluoromethylating reagent

2015 年, Stephenson 小组 ${ }^{[39]}$ 以廉价的三氟乙酸酎为 三氟甲基源, $\mathrm{Ru}(\mathrm{bpy})_{3} \mathrm{Cl}_{2} \bullet 6 \mathrm{H}_{2} \mathrm{O}$ 为光催化剂，吡啶氮氧 盐为氧化剂, 生成的吡啶作为碱, 实现了苯环和杂环的 $\mathrm{C}-\mathrm{H}$ 键三氟甲基化反应(Scheme 19). 该反应中, 三氟 甲基化的区域选择性较差，反应操作简单，且可以放大 到克级规模, 产率中等.

同年, Brase 课题组 ${ }^{[40]}$ 也报道了以三氟乙酸酐为三 
氟甲基源的芳烃或杂环的 $\mathrm{C}-\mathrm{H}$ 键三氟甲基化反应 (Scheme 20). 该反应无需加入金属催化剂, 以室温下易 于储存的过氧化碳酰胺(UHP)为氧化剂, 实现了富电子 的呋喃环、噻吩环、吡咯环、苯环的 $\mathrm{C}-\mathrm{H}$ 键三氟甲基 化反应, 产物产率较低.

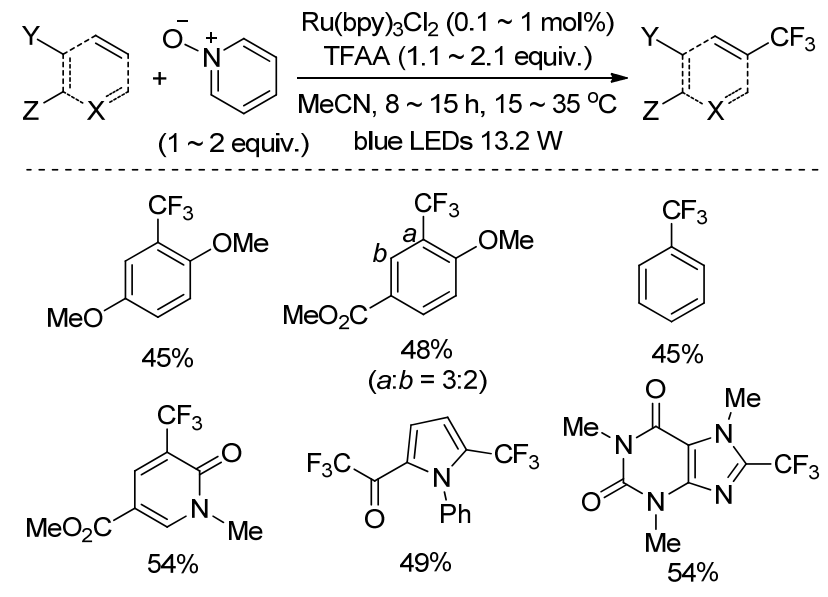

图式 19 规模化且操作简单的自由基三氟甲基化反应 Scheme 19 A scalable and operationally simple radical trifluoromethylation

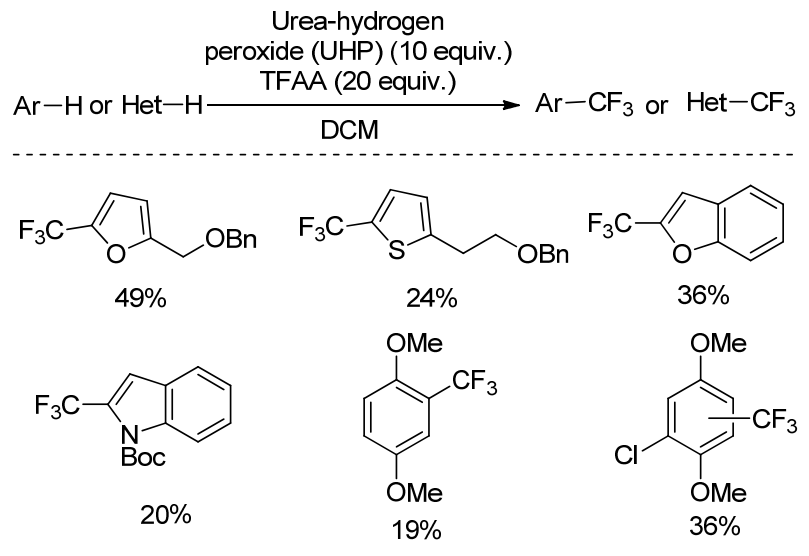

图式 20 无金属参与的(杂)芳烃的自由基三氟甲基化反应 Scheme 20 Metal-free radical trifluoromethylation of (hetero)arenes

2017 年, $\mathrm{Su}$ 课题组 ${ }^{[41]}$ 以 $\mathrm{CF}_{3} \mathrm{CO}_{2} \mathrm{H}$ 为三氟甲基源, $\mathrm{Rh}$ 修饰的 $\mathrm{TiO}_{2}$ 为光催化剂, 催化量的 $\mathrm{Na}_{2} \mathrm{~S}_{2} \mathrm{O}_{8}$ 为氧化剂, 室温反应下实现苯的 $\mathrm{C}-\mathrm{H}$ 键三氟甲基化反应(Scheme 21). 该反应条件温和，底物适用性好，卤素、乙酰基、 酯基、氭基、羧基取代的苯环以及各种氮杂环都能很好 的发生反应, 产率中等至良好. 机理研究表明, 三氟乙 酸分解释放氢气的过程是该反应进行的驱动力, 该反应 经历了自由基反应的历程, 往反应体系中加入自由基捕 捉剂 TEMPO, 成功地捕捉到了三氟甲基自由基.

2018 年, Qing 课题组 ${ }^{[42]}$ 以二(三氟乙酸)五氟碘苯 $\left(\mathrm{C}_{6} \mathrm{~F}_{5} \mathrm{I}\left(\mathrm{OCOCF}_{3}\right)_{2}\right)$ 为三氟甲基源, $\mathrm{Ru}(\mathrm{bpy})_{3}(\mathrm{PF} 6)_{2}$ 为光催
化剂, $\mathrm{CH}_{3} \mathrm{CN}$ 为溶剂, $35{ }^{\circ} \mathrm{C}$ 下反应 $12 \mathrm{~h}$, 实现了含吸电 子基团苯环的 $\mathrm{C}-\mathrm{H}$ 键三氟甲基化反应(Scheme 22). 该 反应条件温和，官能团兼容性好，产率中等至良好，生 成的五氟碘苯可以回收重新制备二(三氟乙酸)五氟碘 苯，提高了反应原子经济性，但三氟甲基化的区域选择 性不高.

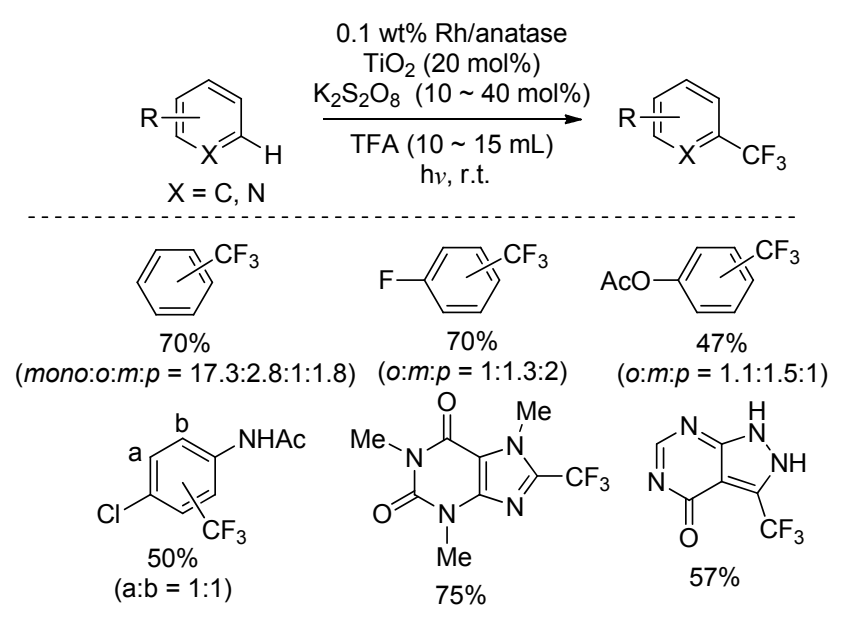

图式 21 光驱动的(杂)芳烃与三氟乙酸的三氟甲基化反应 Scheme 21 Photo-driven trifluoromethylation of (hetero)arenes with trifluoroacetic acid

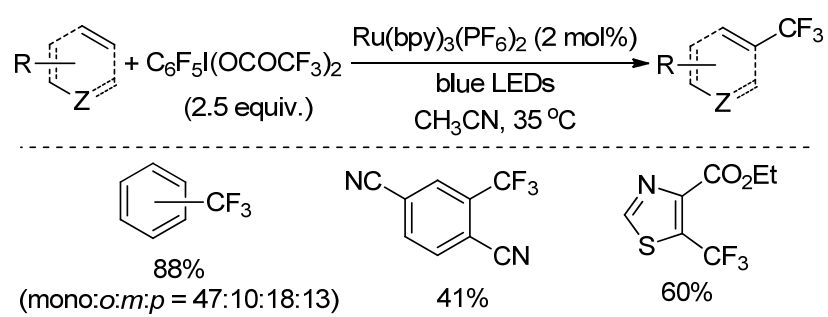<smiles>COc1nc(Cl)nc(OC)c1C(F)(F)F</smiles>

图式 22 (杂)芳烃与三氟乙酸五氟碘苯的脱羧反应 Scheme 22 Decarboxylation of perfluoroarene Iodine(III) trifluoroacetates for trifluoromethylation of (Hetero)arenes

\section{$3 \mathrm{C}=\mathrm{X}(\mathrm{X}=\mathrm{C}, \mathrm{O})$ 的三氟甲基化反应}

通过双键的官能团化反应引入三氟甲基是构建 $\mathrm{sp}^{3}-\mathrm{C}-\mathrm{CF}_{3}$ 键的重要方法之一 ${ }^{[15 \mathrm{a}, 43]}$, 其中包括碳碳双键 和碳氧双键的三氟甲基化反应. 1974 年, $\mathrm{Coe}$ 等 $^{[44]}$ 以现 场生成的 $\mathrm{CF}_{3} \mathrm{CO}_{2} \mathrm{Na}$ 为三氟甲基源, $\mathrm{CH}_{3} \mathrm{CN}$ 和 $\mathrm{H}_{2} \mathrm{O}$ 的混 合液为溶剂, $\mathrm{NaOH}$ 为碱, 利用 Kolbe 电解法实现了烯烃 双键的三氟甲基化反应(Scheme 23). 该反应不能得到 单一的产物, 得到的是多种三氟甲基化的混合产物. 双 键上取代基的电子性质对混合产物的产率影响较大，吸 
电子基团取代的双键反应得到的产率较高, 而给电子基 团取代的双键反应得到的产率较低.

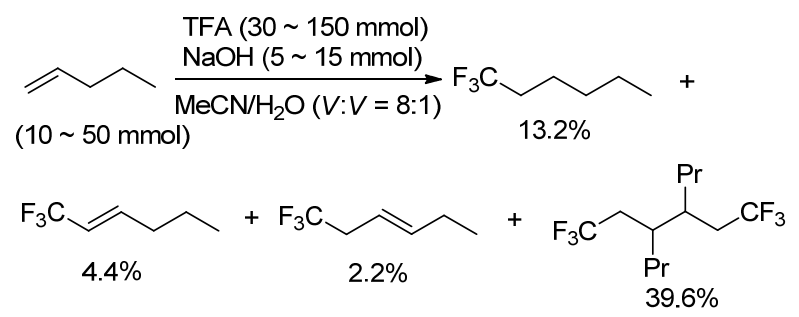

图式 23 烯烃双键与三氟乙酸的三氟甲基化反应

Scheme 23 Trifluoromethylation of olefinic double bonds with trifluoroacetic acid

1986 年, Barton 小组 ${ }^{[45]}$ 以三氟乙酸酐为三氟甲基源, 4-二甲氨基吡啶(DMAP)为催化剂, 乙醚为溶剂, 实现 了 1-着基吡啶-2-硫酮的三氟甲基化反应，生成了 2-三 氟甲硫基吡啶(Scheme 24). 该反应首先在室温下反应 $30 \mathrm{~min}$ 生成相应的酯, 没有被分离出来的酯在铇丝灯下 照射 $15 \mathrm{~min}$, 发生脱羧三氟甲基化反应生成了 2-三氟甲 硫基吡啶. 该反应条件温和，产率较高.

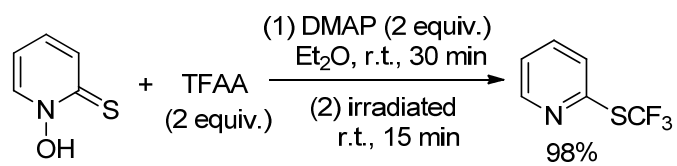

图式 24 1-差基吡啶-2-硫酮的三氟甲基化反应

Scheme 24 Trifluoromethylation of 1-hydroxypyridine-2(1H)thione

2003 年, Langlois 课题组 ${ }^{[46]}$ 以三氟乙酸酯和二取代 三氟乙酰胺为亲核三氟甲基化试剂, DMF 和 THF 的混 合物为溶剂, 在叔丁醇钾作用下室温反应 $24 \mathrm{~h}$, 实现了 二苯甲酮羰基的三氟甲基化反应(Scheme 25). 该反应 条件温和, 只需加入 1 equiv.的三氟甲基化试剂, 产率中 等至良好. 该体系中, 双取代的羰基才能发生亲核三氟 甲基化反应，而单取代的羰基则不能发生三氟甲基化反 应。

2005 年, Chang 小组 ${ }^{[47]}$ 以 $\mathrm{CF}_{3} \mathrm{CO}_{2} \mathrm{Na}$ 为三氟甲基源, $\mathrm{CuI}$ 为促进剂, $\mathrm{DMF}$ 为溶剂, $170{ }^{\circ} \mathrm{C}$ 下反应 $2 \mathrm{~h}$, 实现了 苯甲醛羰基的三氟甲基化反应(Scheme 26), 产率中等 至优秀. 该反应中, DMF 加速了三氟甲基阴离子的生成 或者促进了三氟甲基阴离子对羰基的亲核进攻.

2014 年, Wirth 小组 ${ }^{[48]}$ 同样基于 Kolbe 电解法, 在电 化学微反应器中实现了缺电子烯烃的双三氟甲基化反 应(Scheme 27). 该反应以 $\mathrm{CF}_{3} \mathrm{CO}_{2} \mathrm{H}$ 为三氟甲基源，三 乙胺为碱, $\mathrm{CH}_{3} \mathrm{CN}$ 和 $\mathrm{H}_{2} \mathrm{O}$ 的混合液为溶剂, 在室温下只 需反应 $69 \mathrm{~s}$, 产率中等. 与之前报道的反应相比, 该反 应条件温和、高效。

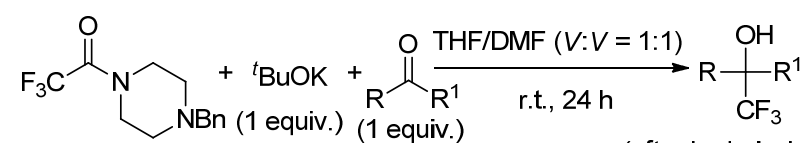

(1 equiv.)

(after hydrolysis)<smiles>OC(c1ccccc1)(c1ccccc1)C(F)(F)F</smiles>

$95 \%$<smiles>OC(c1ccccc1)(c1cccs1)C(F)(F)F</smiles>
$10 \%$<smiles>OC1(C(F)(F)F)c2ccccc2-c2ccccc21</smiles>

$95 \%$<smiles>OC(c1ccccn1)(c1ccccn1)C(F)(F)F</smiles>

$20 \%$<smiles>OC(c1ccccc1)(c1ccccn1)C(F)(F)F</smiles>

$95 \%$<smiles>OC(c1ccccc1)C(F)(F)F</smiles>

$0 \%$
图式 25 三氟乙酸衍生物作为亲核三氟甲基化试剂 Scheme 25 Trifluoroacetic acid derivatives as nucleophilic trifluoromethylating reagents

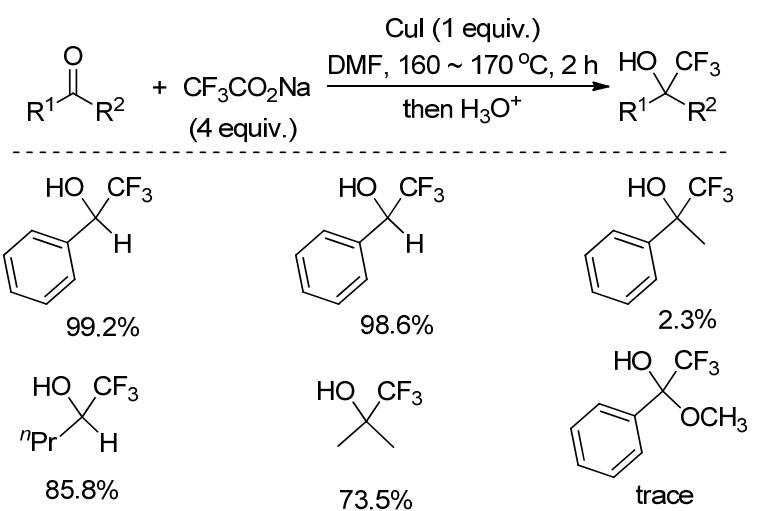

图式 26 碘化亚铜催化的羰基化合物与三氟乙酸钠的三氟甲 基化反应

Scheme 26 CuI-catalyzed trifluoromethylation of carbonyl compounds with sodium trifluoroacetate

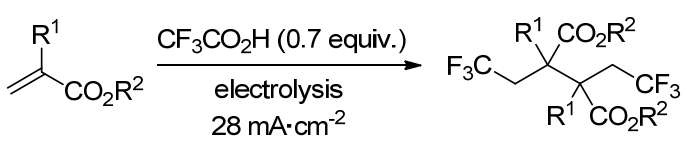

$69 \mathrm{~s}$

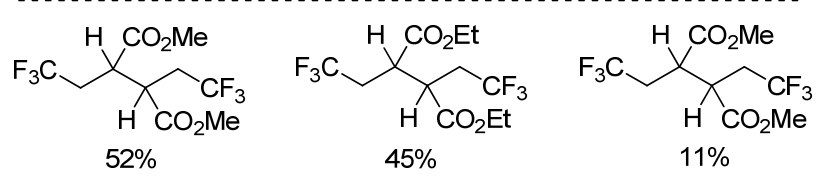

图式 27 烯烃在电化学微反应器中的三氟甲基化反应 Scheme 27 Trifluoromethylation of alkenes in an electrochemical microreactor

2016 年, Sodeoka 课题组 ${ }^{[49]}$ 以三氟乙酸酐(TFAA)为 三氟甲基源，实现了非活化烯丙基的三氟甲基化反应 (Scheme 28). 该反应中, 三氟乙酸酐与过氧化碳酰胺 (UHP)在无金属条件下反应现场生成过氧化物，然后往 体系中加入 $\left[\mathrm{Cu}\left(\mathrm{CH}_{3} \mathrm{CN}\right)_{4}\right] \mathrm{PF}_{6}$ 催化剂和底物. 该反应体 系能够容忍羟基、乙酰基等活性基团，底物适用性广， 
产物产率较高.

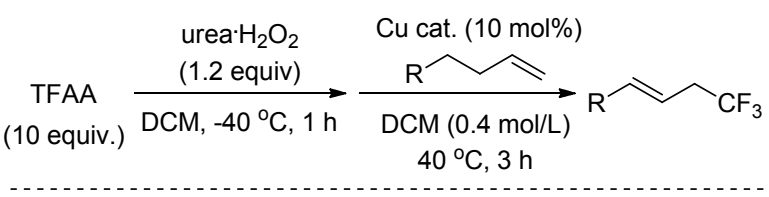<smiles>FC(F)(F)C/C=C/Cc1ccccc1</smiles>

$(E / Z=80: 20)$<smiles>COc1ccc(CC/C=C/CC(F)(F)F)cc1</smiles>
$(E / Z=81: 19)$<smiles>O=C1CCCCC1C=CCC(F)(F)F</smiles>

$(E / Z=81: 19)$<smiles>CC(=O)NCC=CCC(F)(F)F</smiles>

$(E / Z=79: 21)$<smiles></smiles>

$(E / Z=72: 28)$<smiles>CC(CBr)CC=CCC(F)(F)F</smiles>

$(E / Z=80: 20)$
图式 28 惰性烯烃与酸酐的三氟甲基化反应

Scheme 28 Trifluoromethylation of unactivated alkenes with acid anhydrides

2018 年, Sodeoka 课题组 ${ }^{[50]}$ 同样以三氟乙酸酐 (TFAA)为三氟甲基源, 实现了无金属条件下烯烃的三 氟甲基化反应(Scheme 29). 该反应中, 三氟乙酸酐与过 氧化碳酰胺(UHP)在 $0{ }^{\circ} \mathrm{C}$ 下反应 $1 \mathrm{~h}$ 生成过氧化物, 然 后往体系中加入烯烃, $40{ }^{\circ} \mathrm{C}$ 下反应 $1 \mathrm{~h}$, 生成三氟乙酰 氧化三氟甲基化或胺化三氟甲基化的双官能化产物. 机 理研究表明, 烯烃作为单电子转移的给电子体加速了三 氟甲基自由基的产生过程.

此外, 还可以通过三氟乙酸衍生物羰基的缩合反应 引入三氟甲基. 2018 年, Wang 小组 ${ }^{[51]}$ 通过三氟乙酸酐与 胺基的缩合反应合成了含三氟甲基的 2-氨基噻二唑 (Scheme 30).

\section{4 结论与展望}

本文论述了以三氟乙酸及其衍生物为三氟甲基源 的三氟甲基化反应的研究进展, 对其三种反应类型进行 了归纳和总结, 并就其相关机理予以阐述. 三氟乙酸及 其衍生物具有廉价、易得、稳定及副产物是二氧化碳等 优点, 将其作为三氟甲基源在有机分子中引入三氟甲基 具有广阔的发展前景. 然而, 现已报道的以三氟乙酸及 其衍生物为三氟甲基源的反应存在着一些有待解决的 问题. 首先, $\mathrm{C}-\mathrm{X}(\mathrm{X}=\mathrm{Br}, \mathrm{I})$ 键的三氟甲基化反应虽然 具有产物产率较高、区域选择性较好等优点, 但是底物 需要预官能化, 原子经济性不高, 脱羒过程需要较高的 反应温度. 其次, $\mathrm{C}-\mathrm{H}$ 键三氟甲基化反应和碳碳(碳氧) 双键的三氟甲基化反应虽然存在着原子经济性和步骤 经济性等优点, 但是这类反应普遍存在着产率不高、区

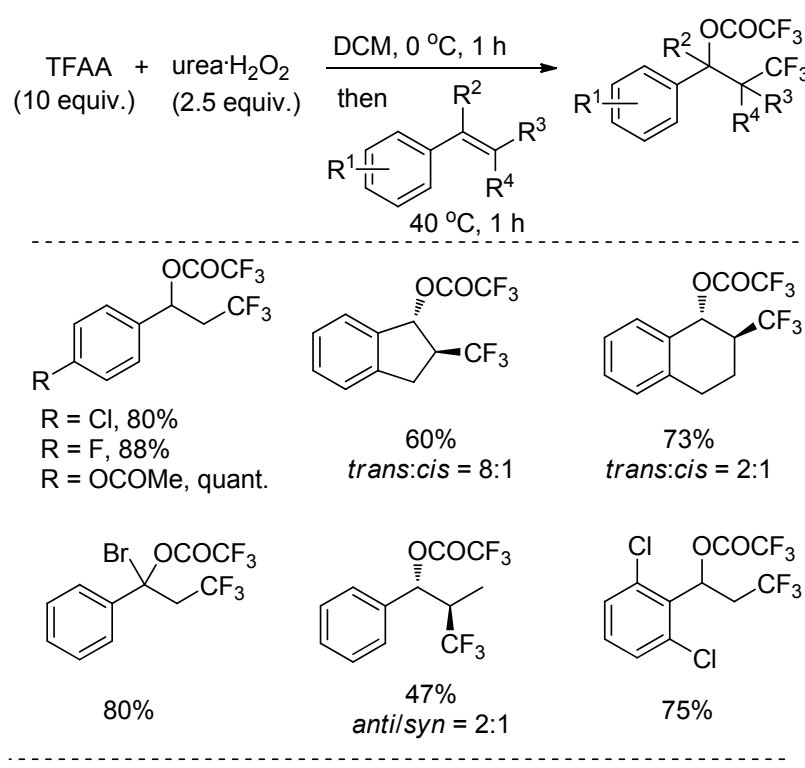

Possible reaction mechanism

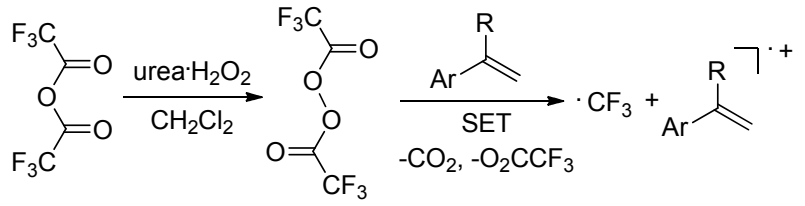

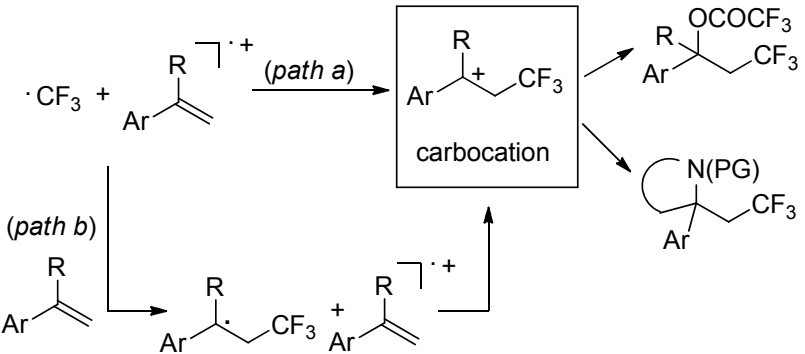

图式 29 无金属条件下烯烃的双官能化

Scheme 29 Metal-free alkene difunctionalization

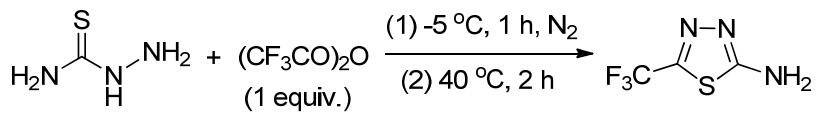

图式 30 合成含三氟甲基的 2-氨基噻二唑

Scheme 30 Synthesis of $\mathrm{CF}_{3}$-containing thiadiazol-2-amine

域选择性不好等缺点，这些也给随后的分离纯化带来了 困难. 对于以三氟乙酸及其衍生物为三氟甲基源的三氟 甲基化反应，如何降低催化剂的使用量和脱羧反应的温 度，如何提高三氟乙酸及其衍生物转化的效率、产物的 产率及区域选择性，将是今后颇具有挑战性的研究方 向.

\section{References}

[1] (a) Hagmann, W. K. J. Med. Chem. 2008, 51, 4359.

(b) Li, Y.; Wu, Y.; Li, G.-S.; Wang, X.-S. Adv. Synth. Catal. 2014, $356,1412$.

[2] Jeschke, P. ChemBioChem 2004, 5, 570. 
[3] (a) O'Hagan, D.; Harper, D. B. J. Fluorine Chem. 1999, 100, 127 (b) Vaillancourt, F. H.; Yeh, E.; Vosburg, D. A.; GarneauTsodikova, S.; Walsh, C. T. Chem. Rev. 2006, 106, 3364.

[4] (a) Müller, K.; Faeh, C.; Diederich, F. Science 2007, 317, 1881. (b) Schlosser, M. Angew. Chem., Int. Ed. 2006, 45, 5432.

[5] Purser, S.; Moore, P. R.; Swallow, S.; Gouverneur, V. Chem. Soc Rev. 2008, 37, 320

[6] (a) Ismail, F. M. D. J. Fluorine Chem. 2002, 118, 27.

(b) Bégué, J.-P.; Bonnet-Delpon, D. J. Fluorine Chem. 2006, 127, 992.

(c) Petrov, V. A. Fluorinated Heterocyclic Compounds: Synthesis, Chemistry, and Applications, John Wiley \& Sons, Inc, Hoboken, NJ, USA, 2009

(d) Ojima, I. Fluorine in Medicinal Chemistry and Chemical Biology, John Wiley \& Sons, Ltd, Chichester, UK., 2009.

(e) Zhu, W.; Wang, J.; Wang, S. J. Fluorine Chem. 2014, 167, 37.

(f) Zhang, J.; Jin, C.; Zhang, Y. Chin. J. Org. Chem. 2014, 34, 662 (in Chinese). (张雯, 金传飞, 张英俊, 有机化学, 2014, 34, 662.)

[7] (a) Penning, T. D.; Talley, J. J. ; Bertenshaw, S.R.; Carter, J. S.; Collins, P. W.; Docter, S.; Graneto, M. J.; Lee, L. F.; Malecha, J. W.; Miyashiro, J. M.; Rogers, R. S.; Rogier, D. J.; Yu, S. S.; Anderson, G. D.; Burton, E. G.; Cogburn, J. N.; Gregory, S. A.; Koboldt, C. M.; Perkins, W. E.; Seibert, K. A.; Veenhuizen, W. Y.; Zhang, Y.; Isakson, P .C. J. Med. Chem. 1997, 40, 1347.

(b) Chakraborti, A. K.; Garg, S. K.; Kumar, R.; Motiwala, H. F.; Jadhavar, P. S. Curr. Med. Chem. 2010, 17, 1563.

[8] (a) Purser, S.; Moore, P. R.; Swallow, S.; Gouverneur, V. Chem. Soc. Rev. 2008, 37, 320 .

(b) Prakash, S. G. K.; Yudin, A. K. Chem. Rev. 1997, 97, 757.

[9] (a) Ye, Y.; Ball, N. D.; Kampf, J. W.; Sanford, M. S. J. Am. Chem Soc. 2010, 132, 14682.

(b) Wang, X.; Truesdale, L.; Yu, J.-Q. J. Am. Chem. Soc. 2010, 132, 3648 .

(c) Zhang, X.; Dai, H.; Wasa, M.; Yu, J.-Q. J. Am. Chem. Soc. 2012, 134, 11948 .

(d) $\mathrm{Mu}, \mathrm{X}$; Chen, S.; Zhen, X.; Liu, G. Chem.-Eur. J. 2011, 17, 6039 .

(e) Zhang, L.-S.; Chen, K.; Chen, G.; Li, B.-J.; Luo, S.; Guo, Q.-Y.; Wei, J.-B.; Shi, Z.-J. Org. Lett. 2013, 15, 10.

(f) Miura, M.; Feng, C.-G.; Ma, S.; Yu, J.-Q. Org. Lett. 2013, 15, 5258 .

(g) Culkin, D. A.; Hartwig, J. F. Organometallics 2004, 23, 3398

(h) Grushin, V. V. Acc. Chem. Res. 2010, 43, 160.

(i) Hughes, R. P.; Meyer, M. A.; Tawa, M. D.; Ward, A. J.; Williamson, A.; Rheingold, A. L.; Zakharov, L. N. Inorg. Chem. 2004, 43, 747.

(j) Ball, N. D.; Gary, J. B.; Ye, Y.; Kampf, J. W.; Sanford, M. S. J. Am. Chem. Soc. 2011, 133, 7577.

(k) Ball, N. D.; Kampf, J. W.; Sanford, M. S. J. Am. Chem. Soc. 2010, 132, 2878.

(1) Cho, E. J.; Senecal, T. D.; Kinzel, T.; Zhang, Y.; Watson, D. A.; Buchwald, S. L. Science 2010, 328, 1679.

[10] (a) Shimizu, R.; Egami, H.; Nagi, T.; Chae, J.; Hamashima, Y.; Sodeoka, M. Tetrahedron Lett. 2011, 51, 5947.

(b) Zhang, C.-P.; Wang, Z.-L.; Chen, Q.-Y.; Zhang, C.-T.; Gu, Y.-C.; Xiao, J.-C. Angew. Chem., Int. Ed. 2011, 50, 1896.

(c) Liu, T.; Shen, Q. Org. Lett. 2016, 13, 2342.

(d) Chu, L.; Qing, F.-L. J. Am. Chem. Soc. 2012, 134, 1298.

(e) Cai, S.; Chen, C.; Sun, Z.; Xi, C. Chem. Commun. 2013, 49, 4552 .

(f) He, Z.; Tan, P.; Hu, J. Org. Lett. 2016, 18, 72.

(g) Li, X.; Zhao, J.; Zhang, L.; Hu, M.; Wang, L.; Hu J. Org. Lett. 2015, 17, 298.

(h) Gao, X.; Xiao, Y.-L.; Wan, X.; Zhang X. Angew. Chem., Int. Ed. 2018, 57,3187 .

(i) Xu, X.; Chen, H.; He, J.; Xu, H. Chin. J. Chem. 2017, 35, 1665.

[11] (a) Hafner, A.; Stefan, B. Angew. Chem., Int. Ed. 2012, 51, 3713.

(b) Ye, Y.; Lee, S. H.; Sanford, M. S. Org. Lett. 2011, 13, 5464 (c) Loy, R. N.; Sanford, M. S. Org. Lett. 2011, 13, 2548

(d) Seo, S.; Taylor, J. B.; Greaney, M. F. Chem. Commun. 2013, 49 , 6385.

(e) Liu, Y.-R.; Tu, H.-Y.; Zhang, X.-G. Synthesis 2015, 47, 3460.

[12] Kino, T.; Nagase, Y.; Ohtsuka, Y.; Yamamoto, K.; Uraguchi, D.; Tokuhisa, K.; Yamakawa, T. J. Fluorine Chem. 2010, 131, 98.

[13] (a) Nagib, D. A.; MacMillan, D. W. C. Nature 2011, 480, 224

(b) Iqbal, N.; Choi, S.; Ko, E.; Cho, E. J. Tetrahedron Lett. 2012 , $53,2005$.

(c) Kamigata, N.; Fukushima, T.; Yoshida, M. Chem. Lett. 1990 649.

(d) Kamigata, N.; Ohtsuka, T.; Fukushima, T.; Yoshida, M.; Shimizu, T. J. Chem. Soc. Perkin Trans. 1 1994, 1339.

(e) Xie, J.; Yuan, X.; Abdukader, A.; Zhu, C.; Ma, J. Org. Lett 2014, 16, 1768.

[14] Mejía, E.; Togni, A. ACS Catal. 2012, 2, 521.

[15] (a) Studer, A. Angew. Chem., Int. Ed. 2012, 51, 8950. (b) Parsons, A. T.; Buchwald, S. L. Nature 2011, 480, 184

[16] (a) Ji, Y.; Brueckl, T.; Baxter, R. D.; Fujiwara, Y.; Seiple, I. B.; Su, S.; Blackmond, D. G.; Baran, P. S. Proc. Natl. Acad. Sci. U. S. A 2011, 108, 14411 .

(b) Fujiwara, Y.; Dixon, J. A.; O’Hara, F.; Funder, E. D.; Dixon, D. D.; Rodriguez, R. A.; Baxter, R. D.; Herle, B.; Sach, N.; Collins, M. R.; Ishihara, Y.; Baran, P. S. Nature 2012, 492, 95.

(c) Wu, X.; Chu,L.; Qing, F.-L. Tetrahedron Lett. 2013, 54, 249.

(d) Yang, Y.-D.; Iwamoto, K.; Tokunaga, E.; Shibata, N. Chem. Commun. 2013, 49, 5510.

(e) Fennewald, J. C.; Lipshutz, B. H. Green Chem. 2014, 16, 1097. (f) Cui, L.; Matusaki, Y.; Tada, N.; Miura, T.; Uno, B.; Itoh, A. Adv. Synth. Catal. 2013, 355, 2203.

[17] (a) Umemoto, T. Chem. Rev. 1996, 96, 1757.

(b) Prakash, G. K. S.; Hu, J. Acc. Chem. Res. 2007, 40, 921.

(c) Prakash, G. K. S.; Yudin, A. K. Chem. Rev. 1997, 97, 757.

(d) Shibata, N.; Matsnev, A.; Cahard, D. Beilstein J. Org. Chem. 2010, 6,65 .

(e) Chu, L.; Qing, F.-L. Acc. Chem. Res. 2014, 47, 1513.

[18] Shi, G.; Shao, C.; Pan, S.; Yu, J.; Zhang, Y. Org. Lett. 2015, 17, 38.

[19] (a) Lopez, S. E.; Salazar, J. J. Fluorine Chem. 2013, 156, 73.

(b) Rui, S.; Lei, L. Sci. China Chem. 2011, 54, 1670.

(c) Rodríguez, N.; Goossen, L. J. Chem. Soc. Rev. 2011, 40, 5030.

[20] Wang, J.; Sanchez-Rosello, M.; Acena, J. L.; del Pozo, C.; Sorochinsky, A. E.; Fustero, S.; Soloshonok, V. A.; Liu, H. Chem. Rev. 2014, 114, 2432.

[21] Matsui, K.; Tobita, E.; Ando, M.; Kondo, K. Chem. Lett. 1981, 12, 1719.

[22] Hünig, S.; Bau, R.; Kemmer, M.; Meixner, H.; Metzenthin, T.; Peters, K.; Sinzger, K.; Gulbis, J. Eur. J. Org. Chem. 1998, 2, 335.

[23] Austin, N. E.; Avenell, K. Y.; Boyfield, I.; Branch, C. L.; Hadley, M. S.; Jeffrey, P.; Johnson, C. N.; Macdonald, G. J.; Nash, D. J.; Riley, G. J.; Smith, A. B.; Stemp, G.; Thewlis, K. M.; Vong, A. K. K.; Wood, M. D. Bioorg. Med. Chem. Lett. 2001, 11, 685.

[24] Langlois, B. R.; Roques, N. J. Fluorine Chem. 2007, 128. 1318.

[25] McReynolds, K. A.; Lewis, R. S.; Ackerman, L. K. G.; Dubinina, G. G.; Brennessel, W. W.; Vicic, D. A. J. Fluorine Chem. 2010, 131, 1108.

[26] Li, Y.; Chen, T.; Wang, H.; Zhang, R.; Jin, K.; Wang, X.; Duan, C. Synlett 2011, 1713

[27] Schareina, T.; Wu, X.-F.; Zapf, A.; Cotte, A.; Gotta, M.; Beller, M. Top Catal. 2012, 55, 426 .

[28] Chen, M.; Buchwald, S. L. Angew. Chem., Int. Ed. 2013, 52, 11628.

[29] Maleckis, A.; Sanford, M. S. Organometallics 2014, 33, 2653.

[30] Lin, X.; Hou, C.; Li, H.; Weng, Z. Chem.-Eur. J. 2016, 22, 2075.

[31] Torikai, K.; Koga, R.; Liu, X.; Umehara, K.; Kitano, T.; Watanabe, K.; Oishi, T.; Noguchi, H.; Shimohigashi, Y. Bioorg. Med. Chem. 2017, 25, 5216.

[32] (a) Giri, R.; Shi, B.-F.; Engle, K. M.; Maugel, N.; Yu, J.-Q. Chem. Soc. Rev. 2009, 38, 3242

(b) Sun, C.-L.; Li, B.-J.; Shi, Z.-J. Chem. Rev. 2011, 111, 1293. 
(c) Liu, C.; Yuan, J.; Gao, M.; Tang, S.; Li, W.; Shi, R.; Lei, A. Chem. Rev. 2015, 115, 12138.

(d) Song, G.; Li, X. Acc. Chem. Res. 2015, 48, 1007.

(e) Guo, X.-X.; Gu, D.-W.; Wu, Z.; Zhang, W. Chem. Rev. 2015, 115,1622 .

(f) Zheng, C.; You, S.-L. RSC Adv. 2014, 4, 6173.

(g) Ackermann, L. Chem. Rev. 2011, 111, 1315.

(h) Neufeldt, S. R.; Sanford, M. S. Acc. Chem. Res. 2012, 45, 936.

(i) Zhang, Y.; Shi, G.; Yu, J.-Q. Carbon-Carbon $\sigma$-Bond Formation via $C-H$ Bond Functionalization in Comprehensive Organic Synthesis, 2nd ed., Vol. 3, Eds.: Molander, G.; Knochel, P., Elsevier, Oxford, 2014, pp. 1101 1209.

[33] Grinberg, V. A.; Polishchuk, V. R.; German, L. S.; Kanevskii, L. S.; Vassiliev, Y. B. Izv. Akad. Nauk SSSR, Ser. Khim. 1978, 3, 673.

[34] Sawada, H.; Nakayama, M.; Yoshida, M.; Yoshida, T.; Kamigata, N. J. Fluorine Chem. 1990, 46, 423.

[35] Lai, C.; Mallouk, T. E. J. Chem. Soc., Chem. Commun. 1993, 17, 1359 .

[36] Matsui, M.; Kondoh, S.; Shibata, K.; Muramatsu, H. Bull. Chem. Soc. Jpn. 1995, 68, 1042.

[37] Tanabe, Y.; Matsuo, N.; Ohno, N. J. Org. Chem. 1988, 53, 4583.

[38] Depecker, C.; Marzouk, H.; Trevin, S.; Devynck, J. New J. Chem. 1999, 23, 739 .

[39] Beatty, J. W.; Douglas, J. J.; Cole, K. P.; Stephenson, C. R. J. Nat. Commun. 2015, 6, 7919.
[40] Zhong, S.; Hafner, A.; Hussal, C.; Nieger, M.; Brase, S. RSC Adv. 2015, 5, 6255 .

[41] Lin, J.; Li, Z.; Kan, J.; Huang, S.; Su, W.; Li, Y. Nat. Commun. 2017, 8, 1 .

[42] Yang, B.; Yu, D.; Xu, X.-H.; Qing, F.-L. ACS Catal. 2018, 8, 2839.

[43] (a) Parsons, A. T.; Buchwald, S. L. Angew. Chem., Int. Ed. 2011, $50,9120$.

(b) Xu, J.; Fu, Y.; Luo, D.-F.; Jiang, Y.-Y.; Xiao, B.; Liu, Z.-J.; Gong, T.-J.; Liu, L. J. Am. Chem. Soc. 2011, 133, 15300.

(c) Wang, X.; Ye, Y.; Zhang, S.; Feng, J.; Xu, Y.; Zhang, Y.; Wang, J. J. Am. Chem. Soc. 2011, 133, 16410.

(d) Liu, X.; Xiong, F.; Huang, X.; Xu, L.; Li, P.; Wu, X. Angew. Chem., Int. Ed. 2013, 52, 6962.

[44] Brookes, C. J.; Coe, P. L.; Owen, D. M.; Pedler, A. E.; Tatlow, J. C. J. Chem. Soc., Chem. Commun. 1974, 9, 323.

[45] Barton, D. H. R.; Lacher, B.; Zard, S. Z. Tetrahedron 1986, 42, 2325.

[46] Jablonski, L.; Joubert, J.; Billard, T.; Langlois, B. R. Synlett 2003, 230.

[47] Chang, Y.; Cai, C. J. Fluorine Chem. 2005, 126, 937.

[48] Arai, K.; Watts, K.; Wirth, T. ChemistryOpen 2014, 3, 23.

[49] Kawamura, S.; Sodeoka, M. Angew. Chem., Int. Ed. 2016, 55, 8740.

[50] Valverde, E.; Kawamura, S.; Sekine, D.; Sodeoka, M. Chem. Sci. 2018, 9, 7115 .

[51] Zhang, Y.; Li, Z.; Song, H.; Wang, B. Chin. J. Chem. 2018, 36, 635.

(Cheng, F.) 